\title{
Heinrich’s Fourth Dimension
}

\author{
Robert Collins \\ UT School of Public Health, Houston, USA \\ E-mail:buckibooster@yahoo.com \\ Received May 6, 2011; revised May 25, 2011; accepted June 5, 2011
}

\begin{abstract}
In this article, the author uses accident data readily available from the United States Bureau of Labor Statistics to create a New Incident Pyramid, a modern day equivalent of Heinrich's Triangle. This historical data is then combined with generally accepted statistical methods to show that the relationship between incident types first envisioned by Herbert William Heinrich in 1931 has a fourth dimension, time. Using statistical analysis methods derived from both a Binomial distribution and a Poisson distribution, this analysis will show how information derived from these accident summaries can be used to predict potential future events. The obvious conclusion reinforced by this analysis will be that the future date for potentially fatal accidents can only be delayed by focusing on accident prevention strategies that address all incidents without regard for the type of resulting injury. This analysis can be used by safety professionals to predict potential future outcomes for their establishments which can then be used to better communicate the need for improvements in accident prevention programs.
\end{abstract}

Keywords: Heinrich's Triangle, Heinrich's Pyramid, New Incident Pyramid, OSHA Recordable Incidents, Lost Workday Cases, Fatalities, Fatal Accidents

\section{Introduction}

In 1931, Herbert William Heinrich, one of the founding fathers of industrial safety and accident prevention, published the results of a study he performed while working for Traveler's Insurance [1]. His original work revealed that, for every "major injury" resulting from a single accident, there were 29 "minor injuries" resulting from accidents and 300 no-injury accidents. The figure he produced, depicting these ratios, came to be known as Heinrich's Triangle. The development of Heinrich's Triangle followed a study of over 5000 cases. Ultimately, the injuries resulting from these cases were categorized as follows:

- Major Injuries: “Any case that is reported to insurance carriers or to the state compensation commissioner." These would be equal to what OSHA (i.e., Occupational Safety and Health Administration) now defines as a recordable incident case.

- Minor Injuries: "A scratch, bruise, or laceration such as is commonly termed a first-aid case."

- No-Injury Accidents: "An unplanned event involving the movement of a person or an object, ray, or substance (slip, fall, flying object, inhalation, etc.), having the probability of causing personal injury or prop- erty damage."

Heinrich's Triangle became the foundation on which many of today's industrial accident prevention programs are built. Perhaps the most important lesson learned from this foundational work is that the path that leads to the prevention of accidents resulting in major injuries lies in investigating all accidents, including "No-Injury Accidents."

In a recent article [2], this author showed how the relationships among incident types first envisioned by $\mathrm{H}$. W. Heinrich still has relevance today. This was accomplished by updating Heinrich's original triangle using BLS (United States Bureau of Labor Statistics) data from a recent 18-year period (1992-2008) to produce a New Incident Pyramid. This data is summarized in Table 1. This analysis showed that for each fatal work-related accident in private industry during the period indicated, there were approximately 500 lost workday cases and 1,000 recordable incidents, as defined by OSHA (Figure 1). Where Heinrich's original work and this author's recent work analyze past incident records, this article uses these past trends and generally accepted statistical methods [3] in an attempt to predict potential future patterns. The purpose of this article is to present these analyses so that conclusions useful to safety profession- 
Table 1. BLS incident data summary.

\begin{tabular}{|c|c|c|c|c|c|}
\hline \multirow[b]{2}{*}{ Year } & \multirow[b]{2}{*}{$\begin{array}{l}\text { Number of } \\
\text { Fatalities }\end{array}$} & \multicolumn{2}{|c|}{ Lost Workday Cases } & \multicolumn{2}{|c|}{ Recordable Incidents } \\
\hline & & $\begin{array}{l}\text { Number of Lost } \\
\text { Workday Cases }\end{array}$ & $\begin{array}{l}\text { Lost Workday } \\
\text { Case Rate }\end{array}$ & $\begin{array}{c}\text { Number of } \\
\text { Recordable Incidents }\end{array}$ & $\begin{array}{l}\text { Recordable Case } \\
\text { Incident Rate }\end{array}$ \\
\hline 2009 & 3890 & $1,667,400$ & 1.8 & $3,277,700$ & 3.6 \\
\hline 2008 & 4670 & $1,900,800$ & 2.0 & $3,696,100$ & 3.9 \\
\hline 2007 & 5112 & $2,036,000$ & 2.1 & $4,002,700$ & 4.2 \\
\hline 2006 & 5230 & $2,114,600$ & 2.3 & $4,085,400$ & 4.4 \\
\hline 2005 & 5214 & $2,184,800$ & 2.4 & $4,214,200$ & 4.6 \\
\hline 2004 & 5229 & $2,225,000$ & 2.5 & $4,257,300$ & 4.8 \\
\hline 2003 & 5043 & $2,301,900$ & 2.6 & $4,365,200$ & 5.0 \\
\hline 2002 & 4978 & $2,494,300$ & 2.8 & $4,700,600$ & 5.3 \\
\hline 2001 & 5281 & $2,559,100$ & 2.8 & $5,215,600$ & 5.7 \\
\hline 2000 & 5347 & $2,752,100$ & 3.0 & $5,650,100$ & 6.1 \\
\hline 1999 & 5488 & $2,742,800$ & 3.0 & $5,707,200$ & 6.3 \\
\hline 1998 & 5457 & $2,780,700$ & 3.1 & $5,922,800$ & 6.7 \\
\hline 1997 & 5616 & $2,866,200$ & 3.3 & $6,145,600$ & 7.1 \\
\hline 1996 & 5597 & $2,832,500$ & 3.4 & $6,238,900$ & 7.4 \\
\hline 1995 & 5495 & $2,972,100$ & 3.6 & $6,575,400$ & 8.1 \\
\hline 1994 & 5959 & $3,061,000$ & 3.8 & $6,766,900$ & 8.4 \\
\hline 1993 & 5643 & $2,967,400$ & 3.8 & $6,737,400$ & 8.5 \\
\hline 1992 & 5497 & $2,953,400$ & 3.9 & $6,799,400$ & 8.9 \\
\hline
\end{tabular}

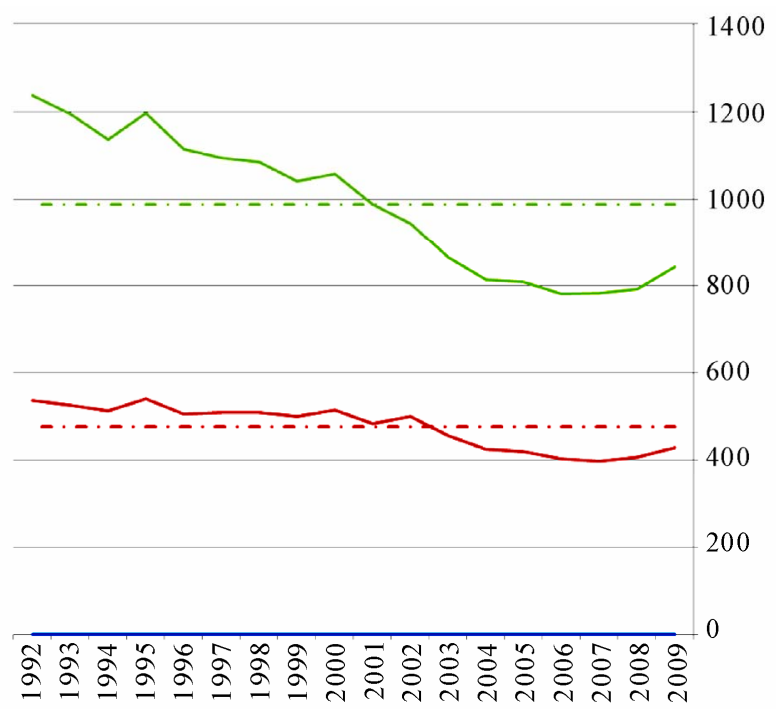

Figure 1. Ratios of lost workday cases and recordable incidents to fatalities.

als can be drawn and used to reduce future incidents. Along the way, additional insights to be used in better communicating the need for improvements in accident prevention programs may be gained.

\section{Methods}

\subsection{Web-Based Injury and Illness Statistics}

Data came from the United States Bureau of Labor Statistics (BLS). BLS provides a variety of annual summaries, including summaries of:

- Fatal occupational injuries by industry and event or exposure. This data is compiled for the Census of Fatal Occupational Injuries (CFOI) using methods described in Chapter 9 of the BLS Handbook of Methods [4];

- Numbers of nonfatal occupational injuries and illnesses by industry and case types. This data is compiled for the Survey of Occupational Injuries and Illnesses (SOII) using methods described in Chapter 9 of the BLS Handbook of Methods [4]; and

- Incident rates of nonfatal occupational injuries and illnesses by industry and case types. This data is also 
compiled for the Survey of Occupational Injuries and Illnesses (SOII) using methods described in Chapter 9 of the BLS Handbook of Methods [4].

It should be noted that these annual summaries are the result of statistical analyses of raw data collected by BLS from representative businesses within the industries mentioned. Access to the underlying raw data is severely restricted. Moreover, the raw data collected by BLS is only a sample collected to represent all businesses/establishments in the United States. Therefore, the data summaries provided by BLS are statistical summaries of samples collected in a manner so as to be representative of all businesses/establishments in the United States.

Without access to the underlying raw data, we cannot know the shape of the data's distribution or the distribution of data for all businesses/establishments in the United States. Without knowledge of the shape of the underlying data, we cannot know whether to use parametric or nonparametric statistical methods to analyze the data in these summaries. Fortunately, the Central Limit Theorem allows to assume that the sample means and confidence limits obtained from summaries of the underlying raw data are normally distributed because the sample size is so very large. Had the underlying raw data been available for analyses, the work presented in this article would have benefited greatly. However, the amount and variety of summary data available from the underlying raw data is sufficient to allow us to perform useful statistical analyses.

\subsection{Data Management}

Trends during 1992-2009 were analyzed to determine the annualized and overall ratios of recordable incidents and lost workday cases to fatal occupational injuries. This data was used to determine the probability of experiencing a fatal occupational injury per incident resulting in an OSHA recordable incident for the time interval analyzed. If we assume that accident prevention programs do not change substantially over the next few years, these probabilities derived from past incidents can be used to predict future trends. With this assumption, probabilities derived from these past incidents are used with generally accepted statistical methods to estimate the future likelihood of fatal accident occurrence.

\subsection{Data Analysis}

The statistical methods chosen for this analysis are those derived from Binomial distributions and Poisson distributions. Binomial distribution methods are used because they represent 1) a series of $n$ independent events or trials, 2) each of which can have only two possible out- comes. In this case, a chronological series of OSHA recordable incidents is the series of $n$ independent events or trials used. "Two events are statistically independent if the probability of their occurring jointly equals the product of their respective probabilities (i.e., $\operatorname{Pr}(A \cap B)=$ $\operatorname{Pr}(A) \mathrm{X} \operatorname{Pr}(B))$." [5] Each independent event is an incident resulting in an OSHA recordable incident that can either result in a fatal or non-fatal injury. As can be seen, a Binomial distribution is suitable for this analysis.

For recordable incidents alone, there are only two possibilities going forward. These are:

1) The recordable incident will result in a fatality; or

2) The recordable incident will not result in a fatality.

These are mutually exclusive and independent events, otherwise known as a Bernoulli Variable. A toss of a coin is a simpler example of a Bernoulli Variable. In each case, the event can take on only 1 of 2 possible values. Each toss of the coin or, in this case, recordable incident represents a single Bernoulli Trial producing a single result. When looking at a string of successive Bernoulli Trials, the appropriate statistical method to use in predicting potential future events of this type is a Binomial distribution. If we wanted to calculate the probability of having $n$ successive trials before encountering $z$ specified outcomes, we would use the following generalized formula:

$$
\text { Probability }=\left[\frac{n !}{z !(n-z) !}\right] p^{z}(1-p)^{(n-z)}
$$

where: $n=$ Total number of consecutive trials or, in this case, recordable incidents without a single fatality;

$z=$ Total number of successes (or failures) or, in this case, one or more fatalities;

$p=$ Probability of a single success or failure (i.e., the probability of one or more fatalities given $n$ consecutive recordable incidents).

Unfortunately, analysis using a Binomial distribution alone gets us only part of the distance to a true temporal probability relationship. In order to establish a temporal probability relationship, a Poisson distribution must be used. Poisson distributions are usually associated with rare events. In this case, the rare event is a fatal occupational incident. Fortunately, Poisson distributions can be used to approximate a Binomial distribution, under certain conditions. Generally accepted statistics theory allows the use of a Poisson distribution to approximate a Binomial distribution when the value of $n$ (i.e., total number of consecutive trials) is large and the value of $p$ (i.e., the probability of a single success or failure) is small. In this case, the value of $p$ is certainly small (i.e., $p$ $=1 / 987=1.01 \times 10^{-3} \pm 8.14 \%$ ) and the value of $n$ increases with time. This means that the approximation of a Binomial distribution using a Poisson distribution im- 
proves with time.

Probability using a Poisson distribution can be calculated using the following formula:

$$
\text { Probability }=\frac{\mathrm{e}^{-\mu} \times \mu^{k}}{k !}
$$

where: $\mu=$ Mean of the Poisson distribution $=\lambda t$;

$\Lambda=$ Number of events per unit time or number of fatalities per unit time;

$t=$ Selected time interval;

$k=$ Total number of successes (or failures) or, in this case, one or more fatalities.

Microsoft Excel and Stata/IC software (version 11, StataCorp LP, College Station, Texas, USA) were used for all of the statistical data analyses presented in this paper. An alpha of $p<0.05$ was selected as the level of significance.

\section{Results}

BLS data for all private industry has been extracted and summarized in Table $\mathbf{1}$ from on-line summary tables. When this data is normalized to the number of fatalities, Figure 1 was created to show the ratio of recordable incidents and lost workday cases to fatalities along with the average values over the 18 -year period shown. The means and $95 \%$ confidence intervals for these ratios are shown in Table 2. Over the 18-year interval studied, there were an average of 987 recordable incidents and 476 lost workday cases for each fatality. If we assume these ratios to be predictive as well as historical, we can use them to determine the probability of future private industry fatalities. The $95 \%$ confidence intervals (as percents of the mean, shown in Table 2) can be considered to be error associated with the probabilities we calculate.

In this case, we will use the Binomial distribution formula presented above to determine the probability of having $n$ successive OSHA recordable incidents without a single fatality. In order to determine this probability, we must first determine the probability of having $n$ successive OSHA recordable incidents without a single success or failure (i.e., zero fatalities) using the single event probability provided above (i.e., $p=1 / 987=1.01 \times$ $10^{-3} \pm 8.14 \%$ ). This simplifies the formula as follows:

$$
\text { Probability }_{\text {zero fatalities }}=\left(1.01 \times 10^{-3}\right)^{0}[0.999]^{(n-0)}
$$

As this is the probability of having zero fatalities in $n$ successive OSHA recordable incidents, the probability of having one (1) or more fatalities in $n$ successive OSHA recordable incidents can be determined using the following formula:

$$
\begin{aligned}
& \text { Probability }_{\text {one (1) or more fatalities }} \\
& =1-\text { Probability }_{\text {zero fatalities }}=1-\left(1.01 \times 10^{-3}\right)^{0}[0.999]^{(n-0)}
\end{aligned}
$$

Collectively, these formulas produce the graph shown in Figure 2. The $x$ axis in Figure 2 represents the number of successive OSHA recordable incidents. In this graph, the solid line represents the probability of having $n$ successive OSHA recordable incidents without having a single fatality (i.e., zero fatalities). The dashed line is the statistical complement to this (i.e., 1 - Probability $_{\text {zero }}$ fatalities) showing the probability of having 1 or more fatalities in $n$ successive OSHA recordable incidents. The lighter lines above and below both of these lines represent the upper and lower bounds, given the error produced at the $95 \%$ confidence level (i.e., $\alpha=0.05$ ). As

\begin{tabular}{|c|c|c|c|c|}
\hline & \multicolumn{4}{|c|}{ 95\% Confidence Limits } \\
\hline & Mean & Lower Limit & Upper Limit & \% Error \\
\hline \multicolumn{5}{|c|}{ All Private Industry: } \\
\hline Ratio of OSHA Recordable Incidents to Fatalities & 987 & 906 & 1,067 & $\pm \mathbf{8 . 1 4}$ \\
\hline Ratio of OSHA Lost Workday Cases to Fatalities & 476 & 451 & 502 & \pm 5.27 \\
\hline \multicolumn{5}{|c|}{ NAICS Code 238 (Specialty Trade Contractors Industry Group): } \\
\hline Ratio of OSHA Recordable Incidents to Fatalities & 442 & 407 & 477 & \pm 7.83 \\
\hline Ratio of OSHA Lost Workday Cases to Fatalities & 221 & 209 & 232 & \pm 5.11 \\
\hline \multicolumn{5}{|c|}{ NAICS Code 211 (Oil and Gas Extraction Industry Group): } \\
\hline Ratio of OSHA Recordable Incidents to Fatalities & 167 & 142 & 191 & \pm 14.4 \\
\hline Ratio of OSHA Lost Workday Cases to Fatalities & 93 & 76 & 109 & \pm 17.4 \\
\hline
\end{tabular}
can be seen, the probability of having $n$ successive OSHA recordable incidents without a single fatality

Table 2. Ratios for all private industry and select industry groups. 


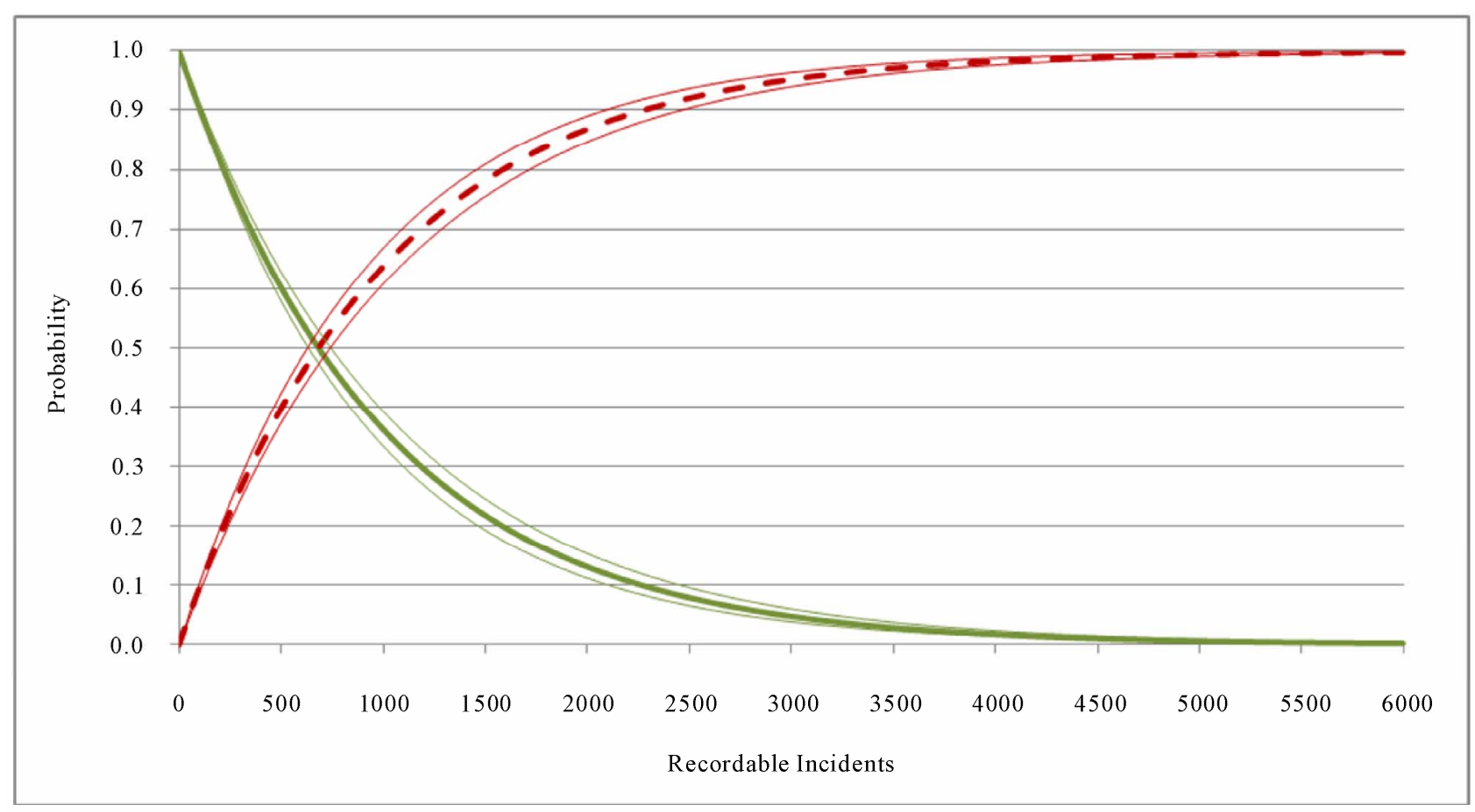

Figure 2. Fatality probabilities per consecutive recordable incident. Upper and lower confience limits (i.e., \% error) are shown as narrower lines above and below.

drops significantly while the probability of having one or more fatalities in $n$ successive OSHA recordable incidents climbs exponentially approaching 1 (or 100\%) asymptotically.

In order to use a Poisson distribution to approximate the Binomial distribution results, we have all of these values in the formula provided above except for the value of $\lambda$ (i.e., the number of events per unit time). We do have the value of $p$ (i.e., the probability of a single success or failure per recordable incident), however. We can use the value of $p$ to get the value of $\lambda$ if we know the temporal frequency of recordable incidents. The formula for determining the value of $\lambda$ from the value of $p$, given the temporal frequency of recordable incidents $(f)$, is:

$$
\lambda=p \times f
$$

where: $\lambda=$ Number of events per unit time

$p=$ Probability of a single success or failure per recordable incident

$f=$ Temporal frequency of recordable incidents per unit of time

The astute reader will recognize that the temporal frequency of recordable incidents is directly related to size of the business (i.e., the number of hours worked each year). It may also be a function of the less quantifiable variables of 1) intrinsic risk of the business; and 2) quality of the accident prevention program.
For the purposes of this example, let's assume that the value of $f$ is 100 recordable incidents per year. Substituting into the formula above, we get a value for $\lambda$ equal to:

$$
\begin{aligned}
\lambda & =\frac{\left(1.01 \times 10^{-3}\right) \text { Fatalities }}{\text { Re cordable Incident }} \times \frac{(100 \text { Re cordable Incidents })}{\text { Year }} \\
& =\left(1.01 \times 10^{-1}\right) \frac{\text { Fatalities }}{\text { Year }}
\end{aligned}
$$

Substituting this value for $\lambda$ into the probability function above yields the following results:

Probability $=$

$$
\frac{\mathrm{e}^{-\left(1.01 \times 10^{-1} \text { fatalities } / \text { Year }\right) \times t} \times\left[\left(1.01 \times 10^{-1} \text { fatalities } / \text { Year }\right) \times t\right]^{k}}{k !}
$$

Figure 3(a) shows the results of this equation graphically. In this case, the $\mathrm{x}$ axis is time in years. The solid line represents the probability of having zero (0) fatali-

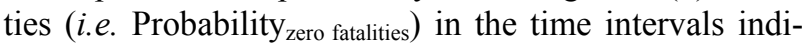
cated in years. The dashed line is the statistical comple-

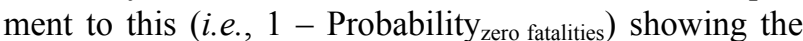
probability of having 1 or more fatalities (i.e., Probabili-

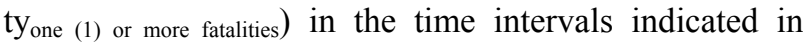
years. The lighter lines above and below both of these lines represent the upper and lower bounds, given the error produced at the $95 \%$ confidence level (i.e., $\alpha=$ $0.05)$. As can be seen, the probability of having zero (0) 
fatalities drops significantly while the probability of having one or more fatalities in the time intervals indicated in years climbs exponentially approaching 1 (or $100 \%$ ) asymptotically. For this example of 100 recordable incidents per year, the probability of having one or more fatalities exceeds $50 \%$ after only 7 years.

If we were to use a different example, say 10 recordable incidents per year, the graph (Figure 3(b)) would appear the same but the $\mathrm{x}$ axis would reach further into the future. In this example, when the $x$ axis is expanded to 500 years the graph is very similar in appearance. For
10 recordable incidents per year, the probability of having one or more fatalities exceeds $50 \%$ after only 69 years.

\section{Discussion}

It should be noted that the ratios of 477 lost workday cases per fatality and 987 recordable incidents per fatality (i.e., 1:477:987) were derived for all private industry. In reality, private industry within the United States is made up of dozens of business types and thousands of

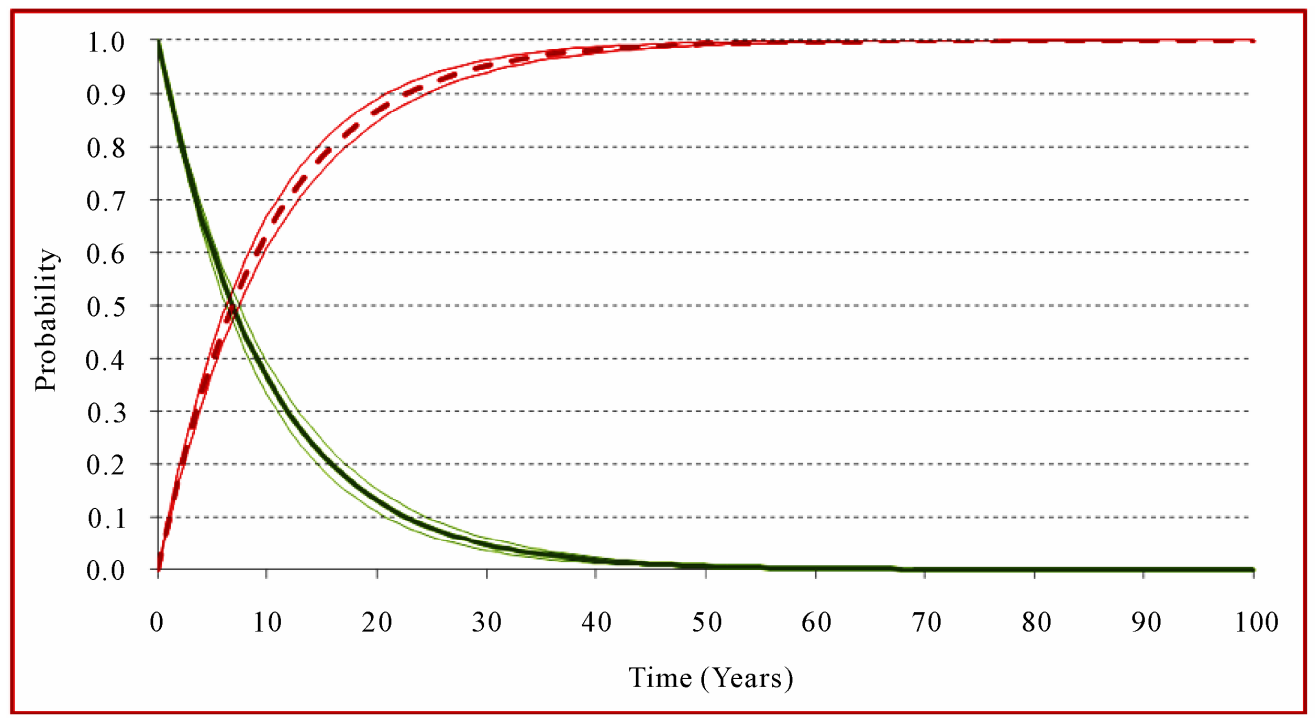

(a)

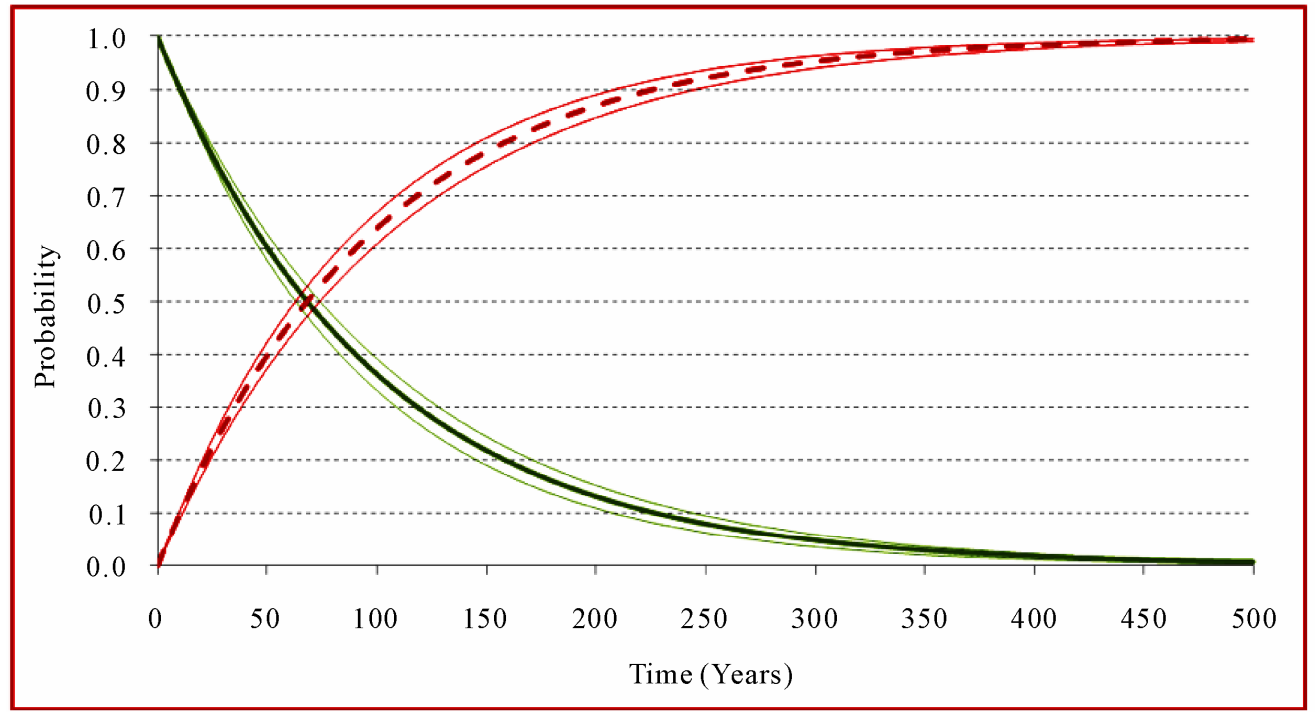

(b)

Figure 3. Fatal probability per year for all Private Industry. Solid line indicates the probability of zero (0) fatalities per year while the dashed line indicates the probability of one (1) or more fatalities per year. Lighter shaded lines above and below represent the upper and lower confidence limits (i.e., \% error). Figure 3(a) assumes a rate of 100 recordable incidents per year. Figure 3(b) assumes a rate of 10 recordable incidents per year. 
individual businesses. It is unlikely that all business types and individual businesses would have the same risk profile. We can see this by looking at two specific examples. In the most recent year for which records are available (i.e., 2009), Specialty Trade Contractors (North American Industrial Classification System or NAICS Code 238) had the highest total number of fatalities (i.e., 477) while Oil and Gas Exploration firms (NAICS Code 211) had among the lowest total number of fatalities (i.e., 17). When we apply the same methodology used above to characterize risk within private industry, we get the risk profiles shown in Table 2. From these results, it can be seen that the risk profile for private industry (i.e., $1: 476: 987)$ is significantly different from the risk profiles for Specialty Trade Contractors (i.e., 1:221:442) and Oil and Gas Exploration (i.e., 1:93:167). These risk profiles have nothing to do with the size of the business (i.e., the number of hours worked each year) and everything to do with those less quantifiable variables of 1) the intrinsic risk of their businesses; and/or 2) the quality of their accident prevention programs.

In any event, different risk profiles mean different probabilities for a single success or failure (i.e., $p$ ) that can be applied as above. The resulting probability graphs (Figure 4(b) for Specialty Trade Contractors and Figure 4(c) for Oil and Gas Exploration), assuming 100 recordable incidents per year, appear very similar to that for all private industry (i.e., Figure 4(a)) with only a shift to the right or left on the $\mathrm{x}$ axis. However, as can be seen, the probability of having one or more fatalities exceeds $50 \%$ after 3 years for Specialty Trade Contractors (NAICS Code 238) and 1 year for Oil and Gas Extraction (NAICS Code 211), assuming an annual rate of 100 recordable incidents per year. This would seem to indicate that working in the Oil and Gas Extraction industry group (NAICS Code 211) is more dangerous (at least from the likelihood of death following an incident) than it is in the Specialty Trade Contractors industry group (NAICS Code 238) and working in either industry group appears to be more dangerous than working in private industry as a whole. This is an especially interesting result, given that two industry groups with diametrically opposite fatality totals during 2009 (i.e., 17 and 477) were chosen. This may be explained by the fact that, based upon hours worked, the Oil and Gas Extraction industry group (NAICS Code 211) is $1 / 10^{\text {th }}$ of the size of the Specialty Trade Contractors industry group (NAICS Code 238 ) and this group is $1 / 100^{\text {th }}$ of the size of all private industry.

This same type of refined analysis can be applied to an individual business/establishment if there is sufficient historical data on which to base a potential future probability. Figure 5 shows how this might be applied to a single business/establishment. The $x$ axis in this figure represents years while the y axis represents probability. The solid line descending from left to right represents the probability of experiencing zero (0) fatal incidents for the general industry type corresponding to a specific business/establishment while the dashed line descending from left to right represents the same probability for a specific business/establishment. The solid line ascending from left to right represents the probability of one (1) or more fatal incidents for the general industry type corresponding to a specific business/establishment while the dashed line descending from left to right represents the same probability for a specific business/establishment. The vertical dashed line represents the current position in time on the graph for this specific business/establishment. Two (2) potentially valuable lessons that can be learned from this figure are:

1) The dashed lines representing a specific business/ establishment are to the left of the solid lines representing the general industry type to which the specific business/establishment belongs. This can be interpreted as meaning that it is more dangerous to work in this specific business/establishment than it is for the group to which this business/establishment belongs.

2) At the specific point in time indicated by the vertical dashed line, the probability that the next OSHA recordable incident will result in a fatal accident is $35.6 \%$ for the specific business/establishment while it would only be $19.9 \%$ for the group to which this business/establishment belongs.

It should be noted, however, that an individual business/establishment will be a subset of the industry group to which it belongs. Moreover, both the Oil and Gas Extraction industry group (NAICS Code 211) and the Specialty Trade Contractors industry group (NAICS Code 238) are subsets of the larger private industry group. While restricting this analysis to a specific industry group or business/establishment might produce more useful results, the confidence interval size (i.e., error) must of necessity increase as well making the results potentially less reliable. Statistically speaking, confidence intervals tend to increase as the sample size decreases. In practice, however, two (2) different businesses within the same industry group may have widely different approaches to accident prevention. A business with limited efforts to prevent accidents, sometimes known as a poor safety culture, may find their accident prediction curve (i.e., the Poisson distribution demonstrated above) shifted to the left compared to the industry group. Whereas, a business with strong efforts to prevent accidents, sometimes known as an excellent safety culture, may find their accident prediction curve shifted to the right. In this example, smaller sample sizes are more 


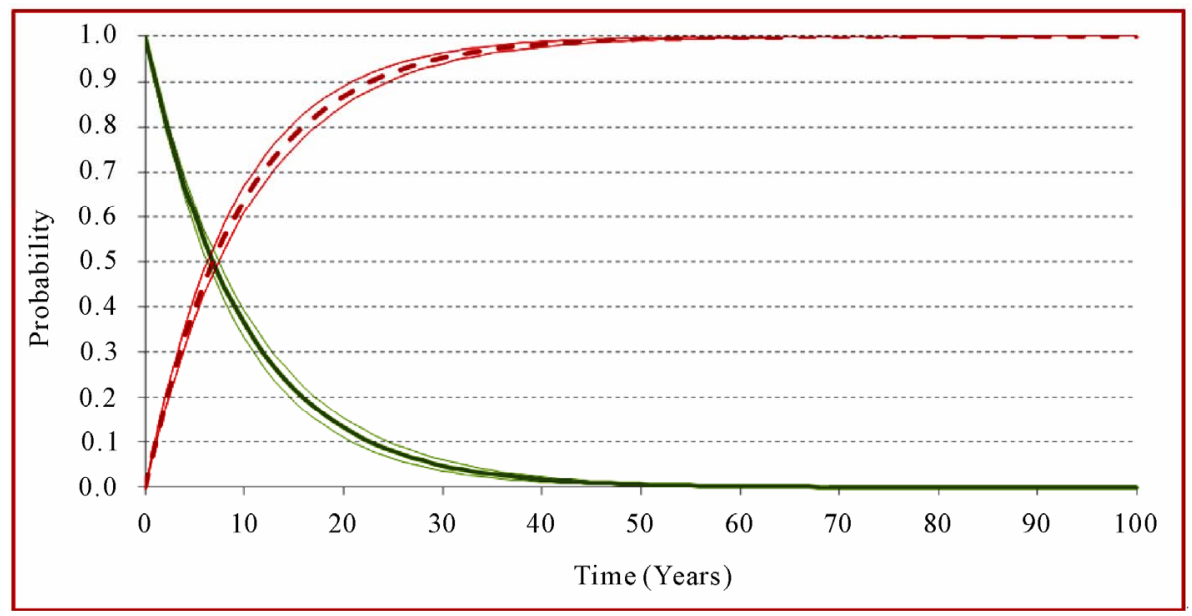

(a)

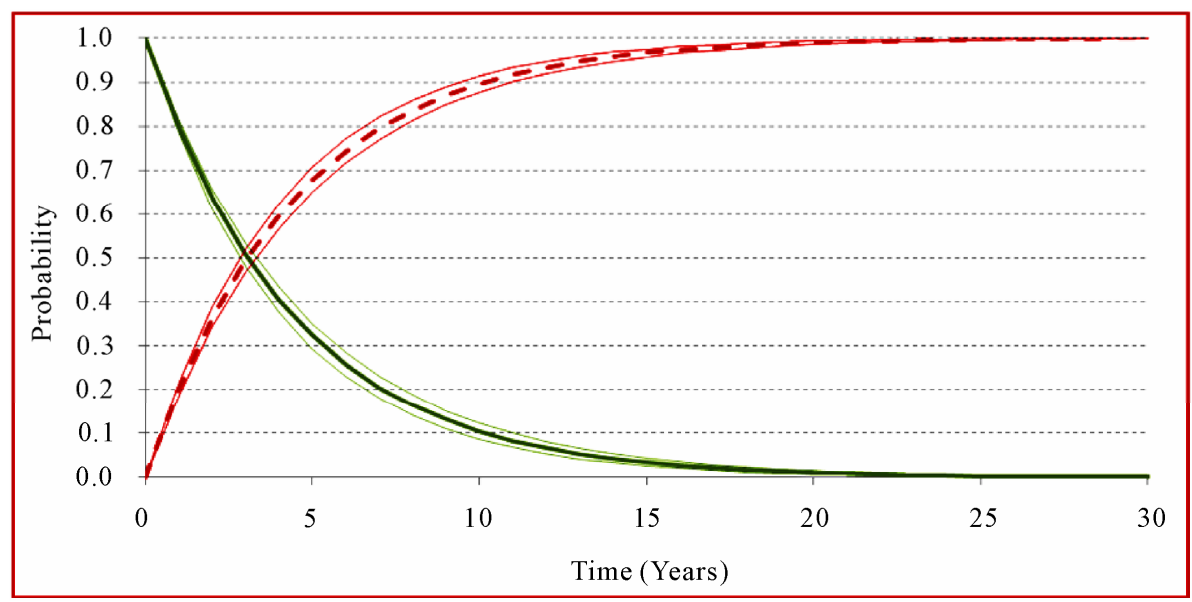

(b)

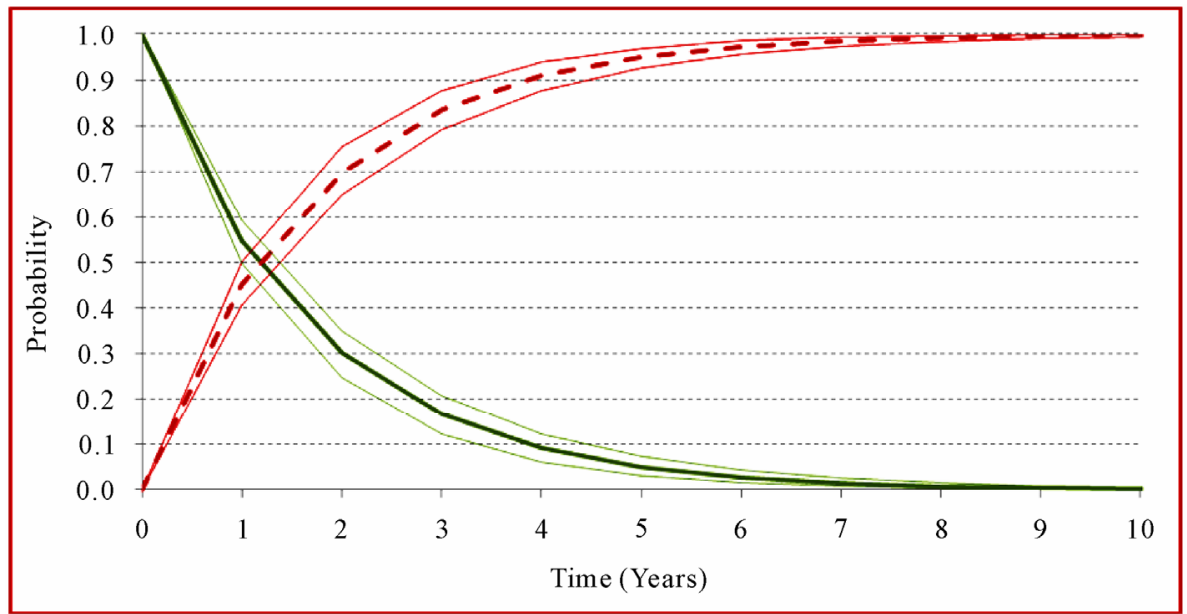

(c)

Figure 4. Fatal probability per year, assuming a rate of 100 recordable incidents per year. Solid lines indicate the probability of zero (0) fatalities per year while the dashed lines indicate the probability of one (1) or more fatalities per year. Lighter shaded lines above and below represent the upper and lower confidence limits (i.e., \% error). Figure 4(a) is for all Private Industry. Figure 4(b) is for the Specialty Trade Contractors industry group (NAICS Code 238). Figure 4(c) is for the Oil and Gas Extraction industry group (NAICS Code 211). 


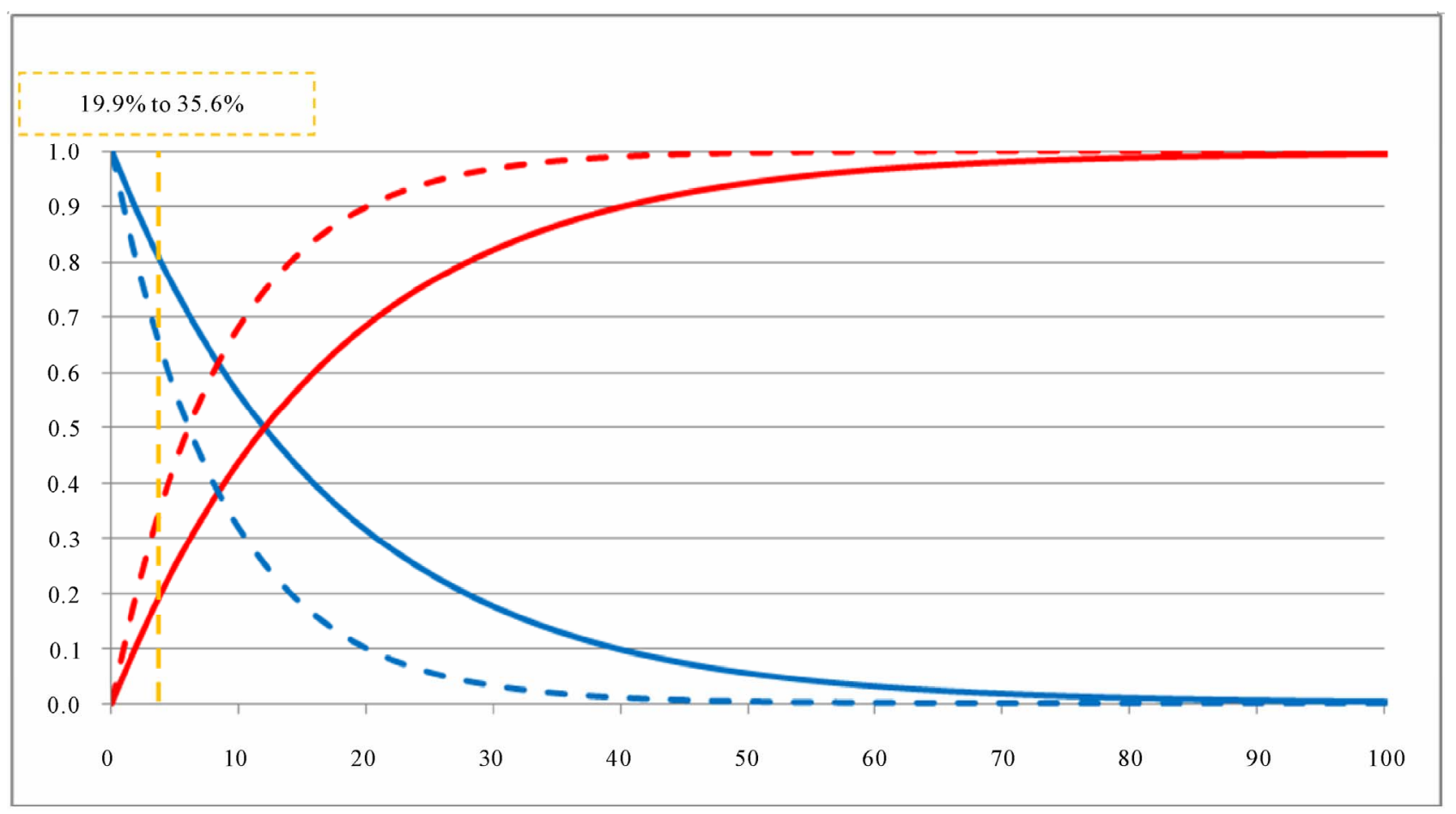

Figure 5. Fatal probability per year for a single business/establishment.

susceptible to variations in the quality of accident prevention policies, programs and procedures.

Readers of Heinrich's original work will note that the three (3) levels of his original triangle consisted of 1) Major Injuries; 2) Minor Injuries; and 3) No-Injury Accidents. The definitions provided for those original three (3) categories were:

1) Major Injuries: "Any case that is reported to insurance carriers or to the state compensation commissioner" [1]. In today's vernacular, these would be OSHA recordable cases.

2) Minor Injuries: "A scratch, bruise, or laceration such as is commonly termed a first-aid case" [1].

3) No-Injury Accidents: "An unplanned event involveing the movement of a person or an object, ray, or substance (slip, fall, flying object, inhalation, etc.), having the probability of causing personal injury or property damage." [1].

From this we can see, that the three (3) levels of the New Incident Pyramid described earlier in this article (i.e., fatalities, lost workday cases and OSHA recordable cases) would all fit into only the top level of Heinrich's original triangle. The bottom two levels of Heinrich's original triangle would appear as two additional layers of this New Incident Pyramid. From Heinrich's original triangle, we know only that these two (2) bottom levels of the New Incident Pyramid would be located below the OSHA recordable case level and that they would be larger than the OSHA recordable case level. Unfortunately, there are no reliable resources to quantify these additional levels. If we were to use the ratios provided in Heinrich's original work, the New Incident Pyramid would appear as seen in Figure 6 for all private industry. If data for these two (2) additional levels of the New Incident Pyramid were more current and reliable, we could use them in the same type of analyses presented in this article to predict potential future fatalities based upon the total number of all incidents instead of only OSHA recordable incidents. As there are no accurate and reliable modern day resources from which data can be gathered for these additional analyses, we can rely only on the analyses presented in this article.

\section{Conclusions}

It should be noted that the statistical analyses presented in this article were performed using summary tables derived from raw data collected by BLS from representative businesses. The statistical analyses performed within this article would have benefitted greatly from access to the underlying raw data in lieu of these summary tables.

One of the fundamental underlying principals of Heinrich's original triangle is that fatalities cannot occur without a foundation of less severe incidents. In other words, increasing numbers of non-serious incidents eventually support the existence of more serious and fatal incidents. We can now see from the analyses presented in this paper that this also applies to incidents 


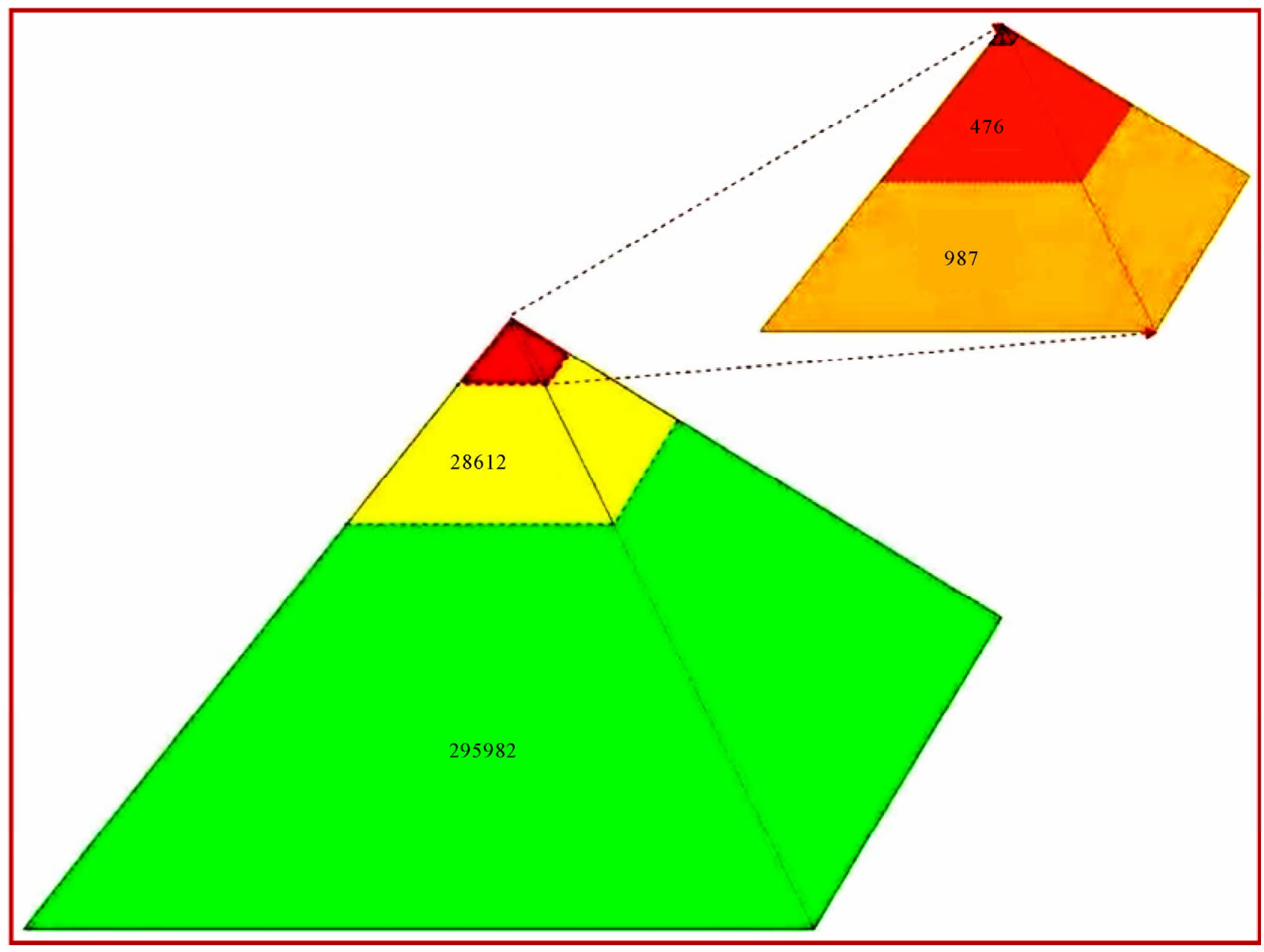

Figure 6. New incident pyramid for all private industry.

occurring over time. Valuable conclusions that can be drawn from this analysis include:

1) Fatal accidents are inevitable. Each day that passes without a fatal occupational accident only leads to an increased likelihood that a fatal occupational accident will occur during the next day as the probability approaches $100 \%$ asymptotically. This conclusion may be dependent on the occurrence of less severe incidents as well as recordable incidents, as demonstrated by the adaption of Heinrich's original triangle shown in Figure 6.

2) Factors potentially affecting when these fatalities will occur include:

a) Size of the business (i.e., number of hours worked each year);

b) Intrinsic risk of the business; and/or

c) Quality of the accident prevention program.

As only one (1) of these factors is within the direct control of business managers, the likelihood of a potential future fatal accident can only be decreased by continually improving the quality of their accident preven- tion programs.

Perhaps the central lesson to be learned from these analyses is that we must always look on recordable incidents (and even accidents without injuries) as portents of fatal accidents to come. Doing little to prevent recordable or minor accidents today will only lead to fatal incidents sooner rather than later; if we do not respond appropriately when they occur. It is only by considering safety and accident prevention with our every thought and action that we can hope to push these fatal accidents further off into the future by shifting our own personal probability distribution to the right.

\section{References}

[1] H. H. William, "Industrial Accident Prevention: A Scientific Approach,"4th Edition, McGraw-Hill Book Company, Boston, 1959.

[2] R. L. Collins, "Heinrich and Beyond," Process Safety Progress, Vol. 30, No. 1, 2011, pp. 2-5.

[3] B. Rosner, "Fundamentals of Biostatistics," Brooks/Cole, 
Pacific Grove, 2010.

[4] United States, "BLS Handbook of Methods," The Bureau, Washington, 1982.
[5] "McGraw-Hill Dictionary of Scientific and Technical Terms," McGraw-Hill, New York, 2003. 